Integrated Kinetic Simulation of

Laser-Plasma Interactions, Fast-Electron Generation and Transport in Fast Ignition

A. Kemp, B. Cohen, L. Divol

November 19, 2009

Physics of Plasmas 
This document was prepared as an account of work sponsored by an agency of the United States government. Neither the United States government nor Lawrence Livermore National Security, LLC, nor any of their employees makes any warranty, expressed or implied, or assumes any legal liability or responsibility for the accuracy, completeness, or usefulness of any information, apparatus, product, or process disclosed, or represents that its use would not infringe privately owned rights. Reference herein to any specific commercial product, process, or service by trade name, trademark, manufacturer, or otherwise does not necessarily constitute or imply its endorsement, recommendation, or favoring by the United States government or Lawrence Livermore National Security, LLC. The views and opinions of authors expressed herein do not necessarily state or reflect those of the United States government or Lawrence Livermore National Security, LLC, and shall not be used for advertising or product endorsement purposes. 


\title{
Integrated kinetic simulation of laser-plasma interactions, fast-electron generation and transport in fast ignition
}

\author{
A.J. Kemp, B.I. Cohen and L. Divol \\ Lawrence Livermore National Laboratory, Livermore, CA
}

(Dated: February 9, 2010)

\begin{abstract}
We present new results on the physics of short-pulse laser-matter interaction of kilojoulepicosecond pulses at full spatial and temporal scale, using a new approach that combines a 3D collisional electromagnetic Particle-in-Cell code with an MHD-hybrid model of high-density plasma. In the latter, collisions damp out plasma waves, and an Ohm's law with electron inertia effects neglected determines the electric field. In addition to yielding orders of magnitude in speed-up while avoiding numerical instabilities, this allows us to model the whole problem in a single unified framework: the laser-plasma interaction at sub-critical densities, energy deposition at relativistic critical densities, and fast-electron transport in solid densities. Key questions such as the multi-picosecond temporal evolution of the laser energy conversion into hot electrons, the impact of return currents on the laser-plasma interaction, and the effect of self-generated electric and magnetic fields on electron transport will be addressed. We will report applications to current experiments.
\end{abstract}

\section{INTRODUCTION}

The availability of intense short petawatt laser pulses at several laboratories around the world allows us to study a host of new physical phenomena and applications of interest for laser fusion, using picosecond pulses with energies of hundreds of Joules at intensities $\gg$ $10^{18} \mathrm{~W} / \mathrm{cm}^{2}$ in the focus [1]. Kinetic modeling of this kind of laser-matter interaction, however, is challenging due to the wide range in temporal and spatial scales that need to be included. While the largest spatial scales are set by the target size, typically sub-millimeter, the smallest scales are determined by the plasma parameters such as the skin length which is a fraction of a laser wavelength in solid density. Similarly, the fastest time scale is determined by the collision frequency in the high density plasma at temperatures below $100 \mathrm{eV}$, which is a fraction of a femtosecond.

The aim of the present work is to provide a computer modeling framework that allows us to simulate the
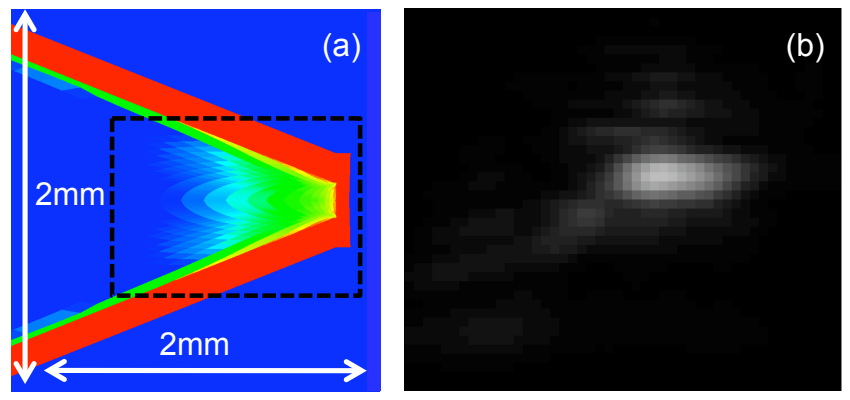

FIG. 1: (Color) Schematic set-up of large cone-target simulation. (a) HYDRA simulation of low-intensity interaction after $1 \mathrm{~ns}$; length scales indicated by white lines, sub-region selected for PIC simulation schematically indicated by dotted black line. (b) Image of aberrated spot used for reconstruction of main pulse in $2 \mathrm{D}$. laser-plasma interaction from vacuum to solid density for short-pulse laser plasma interaction and for fast ignition. A central element of our approach is to use a good approximation to both the preformed plasma expected in the experiment and the footprint of the laser spot. Figure 1 shows an example set-up in the form of a density profile extracted from a three-dimensional hydrodynamic simulation performed with the code HYDRA [2]. Before each simulation we use an electron density map such as the one shown in Fig.1(a) to set up an electron density profile and a map of the charge state of ions. The hydrodynamic simulation is typically run for laser pulse characteristics corresponding to the intrinsic, amplified stimulated emission (ASE) type pre-pulse that is present on most high-power short pulse laser systems, e.g. intensities around $10^{12} \mathrm{~W} / \mathrm{cm}^{2}$ and durations of several nanoseconds. Accounting for the preformed plasma is especially important in cone-shaped targets as they tend to have larger density gradient lengths compared to planar targets, e.g. scale lengths of the order of tens of micrometers, due to the confining effect of the cone walls. It also allows us to make a quantitative assessment of the effect of preformed plasma on the laser absorption.

A second element of our simulations is an accurate representation of the focal spot. The near-field intensity distribution is reconstructed from an image of the highpower focal spot shown in Fig.1(b) to give the boundary conditions for the Maxwell solver in the particle-in-cell (PIC) simulation at the $z=0$ plane of our simulation box. Typically, a realistic focal spot has a multi-peaked intensity distribution which leads to stronger and different filamentation from an ideal Gaussian spot with the same total power.

Finally, the third element of our modeling approach is a description of solid media including resistive effects and ionization. We will present a novel approach that integrates laser-plasma interaction and electron transport in solid matter self-consistently and discuss applications. 


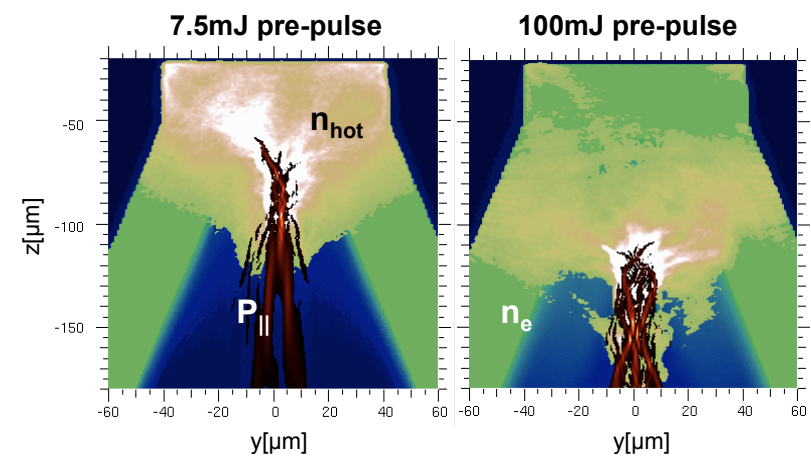

FIG. 2: (Color) PIC simulations of cone interaction with preplasma; shown are Poynting flux along z $\left(P_{\| \mid}\right)$, density of 'cold' electrons $n_{e}$ and 'hot' electrons $n_{\text {hot }}$. (a) Intrinsic pre-plasma case with $7.5 \mathrm{~mJ}$, (b) $100 \mathrm{~mJ}$ pre-plasma case. The laser is incident from below.

\section{STANDALONE CONE}

We have used the set-up procedure described above to model a standalone cone target experiment that was recently performed on Livermore's Titan laser facility [3]. In the experiment, an external pre-pulse with $100 \mathrm{~mJ}$ of energy, an intensity of $10^{12} \mathrm{~W} / \mathrm{cm}^{2}$ and about $3 \mathrm{~ns}$ fwhm was added to the Titan laser's intrinsic ASE pre-pulse, which contains about $7.5 \mathrm{~mJ}$ over $1.7 \mathrm{~ns}$. The key diagnostic was a time-integrated copper K-alpha fluorescence image of the target that was measured with a spherically bent crystal x-ray microscope tuned to the energy of Kalpha emission. The result was that with the additional $100 \mathrm{~mJ}$ pre-pulse, the size of the K-alpha emitting region in the cone increased dramatically while the total energy of K-alpha photons emitted was the same as in a fiducial shot with no external pre-pulse.

To study the physics effects leading to this increased electron divergence with pre-plasma, we have performed large scale 2D kinetic PIC simulations of the cone at full scale, performed with the 3D PIC code PSC [4]. This simulation region comprised an area of $200 \mu \mathrm{m} \times 300 \mu \mathrm{m}$, resolved at 20 cells per micron in each direction, using 50 particles per cell; the simulation time step was $0.07 \mathrm{fs}$ as defined by the Courant condition [7]. The electron density was clamped at $100 n_{c}$, where $n_{c}=1.12 \times 10^{21} \mathrm{~cm}^{-3}$ is the critical density for $\lambda=1 \mu \mathrm{m}$ wavelength laser light, to avoid numerical heating. Figure 2 shows the results of our simulations. Both images combine the hot and cold electron distribution as well as the Poynting flux shown in red below for two configurations, one with the Titan intrinsic prepulse of $7.5 \mathrm{~mJ}$ on the left, and for the case with an external prepulse of $100 \mathrm{~mJ}$ on the right. Note that the image shows only a part of the simulation near the cone tip. Our simulation results suggest that preplasma causes the laser energy deposition to occur upstream in the cone and increases the divergence of the electron beam, as shown in Fig.2. This conclusion is supported by the time-integrated K-alpha images taken in the experiment, which are qualitatively consistent with those generated from our simulation output. They show that in the $100 \mathrm{~mJ}$ pre-pulse case, $\mathrm{K}$-alpha emission is much more widely spread along the cone walls and much weaker at the cone tip than in the $7.5 \mathrm{~mJ}$ case [3].

These simulations allow several conclusions. (1) Standalone cone targets allow only limited conclusions regarding the electron energy spectrum as well as coupling efficiency. This is because the electrostatic confinement of the hot electrons generated in the laser-plasma interaction leads to a strong re-circulation during the picosecond interaction time. While the re-circulating electrons interfere with and actively modify the density and pressure profile near the laser interaction, it is also technically difficult to determine a net energy flux or a spectrum in this kind of simulation set-up; (2) A related problem that is mostly encountered in long-running PIC simulations is associated with particle-absorbing boundaries. When the simulation time exceeds the time for several electron transits in the simulation box, electron currents that accumulate at the box boundaries where they are 'absorbed' lead to a build-up of electrostatic fields, which eventually grow strong enough to reflect $\mathrm{MeV}$ electrons. This affects the simulation result in that it modifies the distribution function and absorption, unless the plasma is surrounded by vacuum, which is the case in our standalone cone simulation; (3) It is important to use a finite mass for the ions rather than ignoring their motion. The typical ion charge states observed in the hydrodynamic set-up correspond to a $Z / A \approx 1 / 2$ ratio between ion charge and mass in the under-dense plasma inside the cone, comparable to that of ionized deuterium. Ions will therefore move significantly over the course of hundreds of femtoseconds. When ion motion is ignored, the electron fluid motion will cause strong non-physical electrostatic fields in the pre-plasma which will alter the result of the simulation.

\section{HYBRID MODEL}

In order to characterize coupling efficiencies and hotelectron spectra in the target we provide more conducting plasma volume to the hot electrons by adding a wire at the tip of the cone. However, we expect the electron transport into the wire to depend on resistive effects in the target. Hence we will need a description of of solid density matter that goes beyond the laser plasma interaction described in the last section. In the following, we will describe briefly our approach which integrates laserplasma interaction and electron transport in solid density media self-consistently.

The key observation that motivates this approach is that transport of laser-generated fast electrons into a solid density target can be reduced to a resistive magneto-hydrodynamic (MHD) description. Figure 3 shows the result of a highly resolved 1D collisional par- 


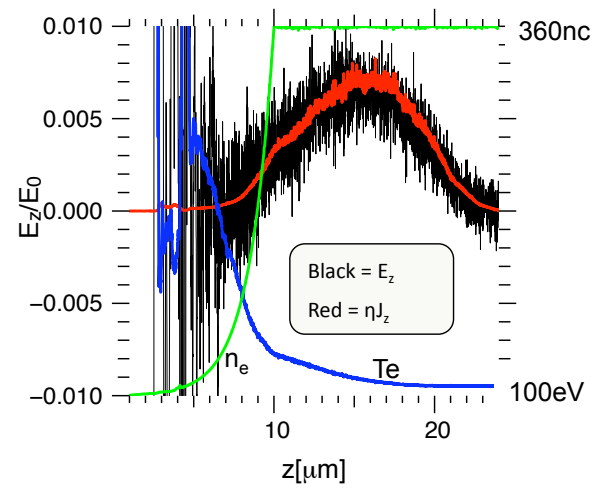

FIG. 3: (Color) Fully resolved collisional PIC simulation result, demonstrating that resistive MHD can be used to describe the fields generated by fast electrons. Shown are lasercycle averages of electron density $n_{e}$, electron temperature $T_{e}$ and longitudinal electric field $E_{z}$, and the value of the Ohmic field $\eta J_{z}$ computed from the current $J_{z}$ and Spitzer resistivity $\eta$, see text, at time $t=93 \mathrm{fs}$.

ticle in cell simulation of a $40 \mathrm{fs}$ fwhm laser pulse interacting with a copper slab. For reasons of simplicity, the charge state of the ions, as well as the Coulomb logarithm used in the collision operator have been set constant to $Z=4$ and $\ln \Lambda=10$, respectively. The solid density slab is preceded by an exponential density gradient with a scale length of $l=1.5 \mu \mathrm{m}$, and it touches the end of the simulation box at $z=25 \mu \mathrm{m}$. We used 200 cells per micron and 100 particles per cell to reduce numerical heating and noise. The main result of this simulation is that there is a good agreement between the electric field from the simulation and the electric field computed from an Ohm's law via a resistivity, defined as

$$
\eta_{\|}=\alpha_{\|}\left(Z^{*}, \mathbf{B}\right) \times \frac{m_{e}}{n_{e} e^{2} \tau_{e i}},
$$

where $\alpha$ is a function of the ionization degree $Z^{*}$ and magnetic field strength $\mathbf{B}, n_{e}$ is the electron density and $\tau_{e i}$ is the electron-ion collision time $[5,6]$.

Following this observation, we have developed a hybrid model that combines our conventional PIC code which solves Maxwells equations with a hybrid model that solves for the electric field from the fast electron current via an Ohm's law

$$
\begin{aligned}
0= & -\mathbf{E}+\eta\left(\mathbf{J}_{e}+\mathbf{J}_{i}\right)-\left(e n_{e}\right)^{-1} \nabla n_{e} T_{e}- \\
& \left(e n_{e} c\right)^{-1} \mathbf{J}_{e} \times \mathbf{B}+\left(e n_{e} c\right)^{-1} \frac{\Delta \mathbf{P}_{c o l l}^{e-f}}{\Delta t},
\end{aligned}
$$

where the thermal current $\left(\mathbf{J}_{e}+\mathbf{J}_{i}\right)$ is determined from the fast electron current $\mathbf{J}_{f}$ via Ampere's law

$$
\mathbf{J}_{e}+\mathbf{J}_{i}=-\mathbf{J}_{f}+\frac{c}{4 \pi} \nabla \times \mathbf{B},
$$

while the magnetic field is solved via Faraday's law in the
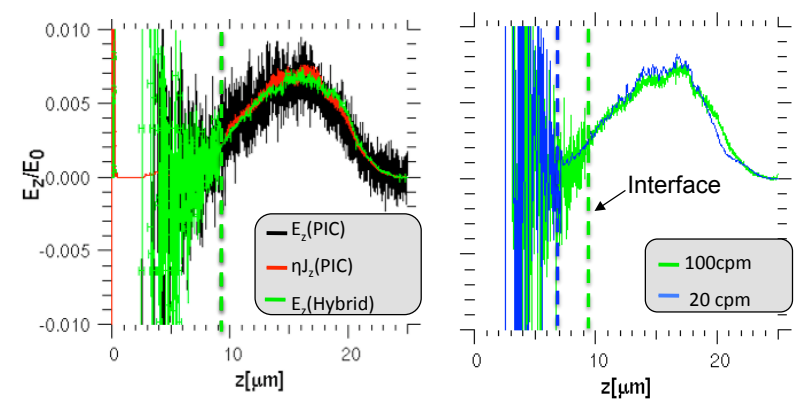

FIG. 4: (Color) PIC vs PIC/Hybrid simulation results for the case discussed in Fig.3 at the same, and reduced resolution. The interface between PIC and Hybrid region is indicated by a dashed line.

same way as in a conventional PIC simulation

$$
\frac{\partial \mathbf{B}}{\partial t}=-c \nabla \times \mathbf{E}
$$

All field- and particle quantities are computed on the same grid, and all particles are treated as in conventional PIC simulations. In particular, the fast-electron current $\mathbf{J}_{f}$ is determined on a particle basis. In Ref. 6 we present a detailed discussion on how the electron density needed in Eq.(2) is computed from the particles with a local drift velocity correction made to ensure consistency with the divergence of Eq.(3). The electron temperature is determined in each cell as $T_{e} \propto\left(\overline{\mathbf{v}}^{2}-\overline{\mathbf{v}}^{2}\right) / 3$ from the cold particle velocities, where the bar symbol stands for the average over all electrons in a cell. Particles with a velocity greater than $5 v_{t h}$, where $v_{t h}=\sqrt{T_{e}}$ is the thermal velocity, are defined as 'fast' and accounted for in $\mathbf{J}_{f}$. As the local temperature evolves, electrons contribute to the fast- or background population depending on their velocity. Collisions play a key role in our hybrid model by (a) thermalizing the electron population; (b) introducing resistivity due to collisions between 'background' electrons and ions; and (c) defining stopping power, as well as scattering of fast electrons off background electron and ions, respectively; in our code we use a collision operator as described in Ref. [8], which is consistent with the expression for resistivity defined in Eq.(1).

Equations (2-4) deliberately exclude the terms that describe the displacement current $-1 /(4 \pi) \partial \mathbf{E} / \partial t$ on the r.h.s. of Eq. (3) and electron inertia $\propto m_{e}(\partial / \partial t+\mathbf{v} \nabla) \mathbf{v}$ on the l.h.s. of Eq. (2). The displacement current can be re-introduced into our equations without loss of explicitness in the solver scheme [6], and does not make a large difference in the simulation results except in situations where the electron currents change rapidly over several femtoseconds, which is typically not the case. The electron inertia term, on the other hand, is essentially responsible for the generation of plasma waves, and thereby causes a numerical instability of the Maxwell solver when the Debye length or the local plasma frequency are not resolved [7]. By ignoring electron inertia, we remove 
time scales proportional to $\omega_{p e}^{-1}$ from the problem. This assumption, which is the common basis of various approaches similar to ours [9-11], is justified under circumstances where (1) the collision frequency becomes comparable to the local plasma frequency, and (2) the density of fast particles is negligible compared to the cold background so that fast particles do not produce a significant charge separation field [12]. Figure 4(a) shows the result of a 1D simulation of the same problem as discussed in Fig. 3, now performed with the PIC/Hybrid algorithm at a resolution of 100 cells per micron. The Ohm's law solver is applied to the plasma on the r.h.s. of the interface indicated by the dashed line. There is good agreement between the electric field in both simulations. Details of this comparison are discussed in Ref. [6].

In this problem the PIC solver is applied to plasma with electron densities below $90 n_{c}$. Since the Hybrid code does not have to resolve the scales determined by plasma physics at high density, we can use a relaxed resolution of 20 cells per micron without risking numerical heating effects in the dense plasma region. Figure 4(r.h.s.) shows the results of PIC and PIC/Hybrid simulations that demonstrate how PIC/Hybrid simulations at 5 times lower resolution give an almost identical result as the runs at the full resolution. As a side remark, note that a conventional collisional PIC simulation at a resolution of 20 cells per micron would give a meaningless result due to numerical self heating and subsequent loss of resistivity in the dense plasma.

In conclusion, we gain a significant computational speed-up by reducing the numerical resolution in our simulations without the penalty of numerical heating at high density. This speed-up factor is more than the expected factor of 25 in our 1D example which comes from the simulation time step being reduced by the same amount as the spatial resolution. In two dimensions, our scheme therefore yields a speed-up of more than 500 making fullscale simulations of experiments possible. As a guideline, the simulation cost for the large conventional 2D PIC simulation of the standalone cone shown in Sec. II was $60,000 \mathrm{~h}$ on 256 cpus of Livermore's Hera cluster. On the other hand, this means that three-dimensional simulations at full scale are still too large for current computers, as they would be approximately 4000 times more expensive than corresponding $2 \mathrm{D}$ runs.

\section{CONE-WIRE}

Cone-wire targets allow us to extract information on coupling efficiency and the electron distribution function beyond what is possible in the standalone cones discussed above. These targets consist of a metal cone with a wire attached at the cone tip. Due to its small density-radius product the wire allows diagnostic access to the emitted $\mathrm{K}$-alpha fluorescence that is related to the hot-electron population, making it an attractive choice for experiments. We expect, however, that electrostatic fields sur-

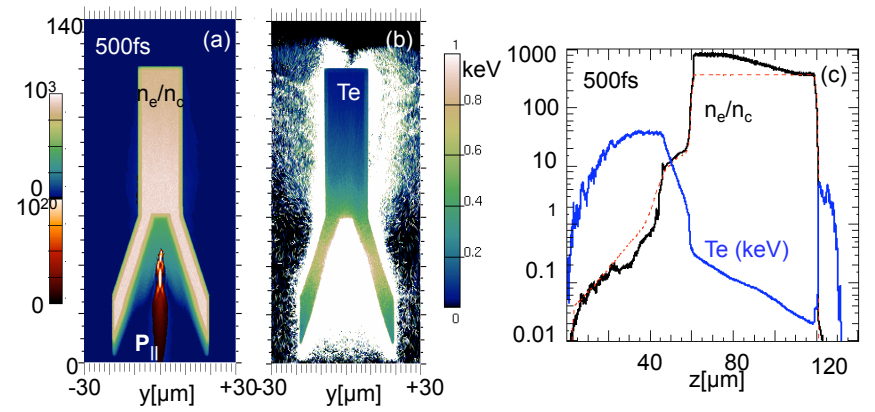

FIG. 5: (Color) Hybrid simulation of cone-wire target. Shown are cold electron density and Poynting flux along z, electron temperature, and line-outs along $y=0$ at $t=500 \mathrm{fs}$. The dotted line in (c) indicates the initial electron density before ionization.

rounding the wire will have a significant effect on the electron trajectories and the subsequent K-alpha emission pattern. In our modeling we therefore need to include the transition between the high-density plasma in the wire, where we apply the 'hybrid' solver, and the vacuum surrounding it. We also expect that the cone tip, as well as the wire, will become ionized by the resistive heating caused by the return currents. This means that we need to include ionization effects to account for the heating of the target material.

To simulate cone-wire targets we have extended our PIC/Hybrid model from a static, planar interface to arbitrarily shaped and temporally dynamic interfaces. When setting up a simulation the interface between the PIC and Hybrid regions is defined by the threshold density, thereby surrounding the entire target. During the simulation run the position of the interface is frequently reset as ionization changes the density profile. Numerically, our algorithm decides for each cell which solver to use for the electric field while the magnetic field in integrated from Faraday's law everywhere.

Ionization of solid copper is included via a simple expression for the effective charge state $Z^{*}\left(T_{e}\right)$ which is interpolated from a Thomas-Fermi equation of state [13]. The electron density is periodically adjusted by determining $Z^{*}$ in each cell and then increasing the weights of thermal electrons, as well as the ion charge state, as appropriate. This is done in an energy-conserving fashion by effectively shrinking the phase space of thermal electrons. We then remove the energy that corresponds to the atomic ionization potentials in the same way. As a result of these operations, the heat capacity of the target material is much higher than in comparable simulations where a fixed charge state is assumed. Since ionization effectively cools the target it can lead to a significant enhancement of resistive effects, compared to cases where ionization is not accounted for.

Figure 5 presents our first simulation results of a conewire target with the PIC/Hybrid code. The cone is a 


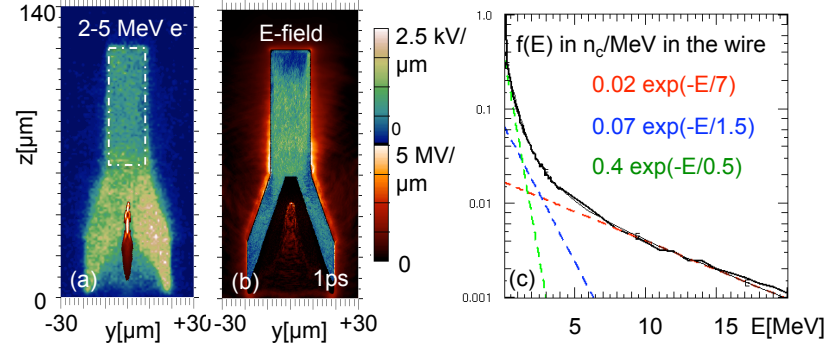

FIG. 6: (Color) Hybrid simulation of cone-wire target; (a) hot-electron density; (b) static electric fields; (c) electron density distribution in wire, as indicated by dashed box in (a), with three slope temperatures fitted as labeled.

1/5-scaled version of that shown in Fig.2, with a $60 \mu \mathrm{m}$ wire attached to the cone tip consisting of copper with an initial charge state $Z^{*}=4$. While the scaling affects some properties of the laser beam propagation in the under-dense plasma which makes quantitative comparisons with experiments impossible, it allows qualitative insights into the physics of cone-wire targets and trends with respect to the amount of pre-plasma which we will explore below. The laser pulse has a Gaussian transverse intensity profile with $5 \mu \mathrm{m}$ fwhm and a Gaussian temporal shape with $300 \mathrm{fs}$ fwhm. At the time $t=500 \mathrm{fs}$, at which the electron density and temperature are shown, the peak laser flux reaches the cone tip. The Poynting flux in Fig. 5(a) shows strong self-focusing into a single filament in which the intensity exceeds twice the value it would have in a vacuum focus. The temperature plot in Fig. 5(b) shows how the divergent hot-electron distribution heats the cone walls near the point of absorption, as well as how the cone tip and the wire are heated resistively. The white regions in the temperature plot correspond to the presence of relativistic particles in vacuum which lead to a large value, rather than an actual temperature. Figure 5(c) shows line-outs of electron density and temperature along the target central axis. An ionization wave driven by resistive heating leads to a significant increase in the electron density, as indicated by original electron density profile shown as the dotted line. Note that the electron density at the point where the laser is absorbed, i.e. at $z=45 \mu \mathrm{m}$ is around $10 n_{c}$. Similar to the standalone cone simulation discussed above, we observe relatively shallow density profiles that do not exceed a value of $50 n_{c}$ even at late times.

Looking at the electron distribution function in the wire, as outlined in Fig.6(a), we observe multiple slopes ranging from the background temperature of $200 \mathrm{eV}$ up to a hot tail with $7 \mathrm{MeV}$, as shown in Fig.6(c). The energy spectrum is given in units of $n_{c} / \mathrm{MeV}$ so that one can directly read off a density associated with the energy, which yields a conversion efficiency. We find that at $1 \mathrm{ps}$, around $90 \%$ of the laser pulse has been absorbed, and about $20 \%$ of the laser pulse energy has been coupled
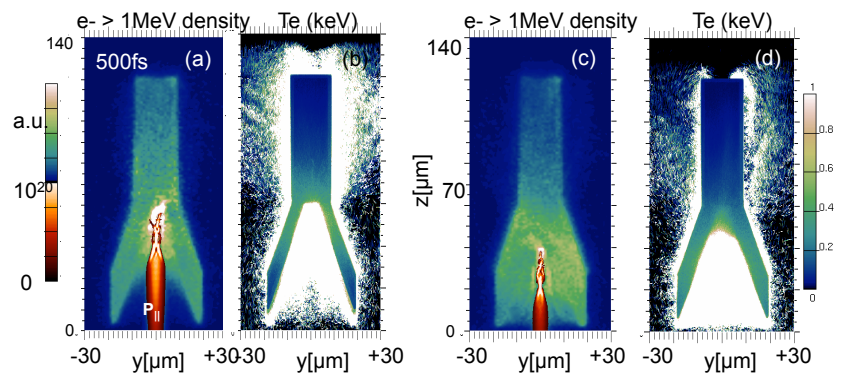

FIG. 7: (Color) Hybrid simulation of cone-wire targets with different levels of pre-plasma. The left case corresponds to $10 \mathrm{~mJ}$ of pre-pulse energy, the right one to $100 \mathrm{~mJ}$. Shown are hot-electron density, Poynting flux (a,c) and temperature (b,d).

into the wire. In particular, about $7 \%$ has been coupled into the background at $200 \mathrm{eV} ; \approx 3 \%$ into intermediate energies $0.1 \mathrm{MeV} \leq E \leq 1.5 \mathrm{MeV}$; and $10 \%$ into electrons with energies $\geq 1.5 \mathrm{MeV}$. As a result we expect that $\mathrm{K}$ alpha emission will be mostly generated by the abundant lower-energy part of the distribution function with energies greater than the K-alpha threshold of $8 \mathrm{keV}$. At the same time we observe that a multi- $\mathrm{MeV} /$ micrometer electrostatic sheath field is built up around the target, as shown in Fig. 6(b), while the interior of the wire is dominated by resistive fields on the order of $1 \mathrm{keV} /$ micron leading to resistive heating and ionization. The sheath field is strong enough to confine most of the $\mathrm{MeV}$ electrons in the wire.

When comparing to a second simulation where the preplasma has been generated by a $100 \mathrm{~mJ}$ pre-pulse, i.e., ten times more energy than in the previous case, we find that the hot-electron energy coupled into the wire is dramatically reduced. Figure 7 shows the results for the number density of electrons with energy $>1 \mathrm{MeV}$ and temperature in two cases, one corresponding to a pre-plasma generated by $10 \mathrm{~mJ}$ of pre-pulse and a second one with a $100 \mathrm{~mJ}$ pre-pulse. Figures $7(\mathrm{a}, \mathrm{c})$ show that (i) laser absorption occurs closer to the cone tip and (ii) hot-electron density in the wire is higher in the $10 \mathrm{~mJ}$ case. Figures $7(\mathrm{~b}, \mathrm{~d})$ show that the wire is hotter while the wings of the cone are colder in the $10 \mathrm{~mJ}$ case, similar to the standalone cone. The conclusion is that with large amounts of pre-plasma the hot electron population generated in the laser-plasma interaction becomes more divergent, and the fraction captured into the wire drops. This agrees qualitatively with recent experimental results on the dependence of K-alpha coupling efficiency from cone-wire targets with respect to the energy in an external pre-pulse [14]. Figure 7 (b,d) also shows some surface heating of the wire, even though our results likely under-estimate this effect. With our PIC/Hybrid scheme it is difficult to model 'infinitely' steep density gradients between under-dense plasma and solid density, because in these regions the displacement current and 
quasi-neutrality become important. In our modeling we have surrounded high-density plasma with a $0.5 \mu \mathrm{m}$ scale length density gradient. This does not modify the electrostatic confinement of energetic electrons because the electrostatic potential depends on the electron distribution in the wire; however, ion acceleration or surface heating effects might be under-estimated, compare Ref. [15]. In a fast-ignition relevant context, on the other hand, we expect no sharp boundaries between the PIC and Hybrid regions.

\section{CONCLUSION}

In performing large-scale kinetic simulations of shortpulse laser interaction with cone targets under realistic conditions, we have gained significant insights into preplasma effects on the laser-plasma coupling and the characteristics of laser-generated hot electrons. We find that an accurate modeling of the pre-pulse is important because the laser-plasma coupling is very sensitive to the presence of pre-plasma which has the effect of moving the point of absorption upstream in the cone, thereby reducing the coupling into the cone tip. Standalone cone targets, however, do not allow us to infer what absorption or transport will be. These aspects are better captured in cone-wire targets, where the coupling into a relatively thin wire provides information on absorption and hot electron transport. Here we find again that pre-plasma significantly reduces coupling into the wire while enhancing the heating of the cone walls further upstream in the target with respect to the laser irradiation direction. Also, there are significant electrostatic potentials arising around the entire target due to multi-MeV electrons that are generated early on in the laser interaction; these potentials are able to confine the hot electrons generated later in the interaction and possibly modify transport in the wire.

To be able to model transport in cone-wire targets over picosecond time scales as required for a meaningful comparison with the experiment, we have extended our existing 3D particle-in-cell code with a resistive MHD module. The key assumption is the neglect of electron inertia, and we solve for the electric field using an Ohm's law. Our approach allows us to model solid density targets with arbitrary geometry and with ionization, which modifies the shape of the interface between the PIC and the MHD regions over time. We are now in a position to evaluate laser-coupling aspects of high-energy petawatt laser experiments and fast-ignition point designs at full-scale in 2D.

A.K. wants to thank S. Wilks for providing HYDRA simulations, D. Hey for providing measurements of the focal spot, and D. Strozzi, M. Tabak, M. Key, H. McLean and P.Patel for helpful discussions and encouragement. This work was supported by the Office of Fusion Energy Sciences. Simulations were carried out on the Livermore Computing Center's Hera cluster under a LLNL Grand Challenge allocation. This work was performed under the auspices of the U.S. Department of Energy by the Lawrence Livermore National Laboratory under Contract DE-AC52-07NA27344.
[1] M Tabak, J Hammer, ME Glinsky, W Kruer, S C Wilks, J Woodworth, E. Michael Campbell, and M Perry. Ignition and high gain with ultrapowerful lasers. Phys Plasmas, 1(5):1626, Nov 1994.

[2] M. M Marinak, G. D Kerbel, N. A Gentile, O Jones, D Munro, S Pollaine, T. R Dittrich, and S. W Haan. Three-dimensional hydra simulations of national ignition facility targets. Phys Plasmas, 8:2275, May 2001.

[3] A.MacPhee et al., Limitation on Pre-pulse Level For Cone-Guided Fast-Ignition ICF, Phys Rev Lett 104:055002 (2010)

[4] H. Ruhl, in Introduction to Computational Methods in Many Body Physics, Ed. M.Bonitz (Rinton Press, New Jersey, 2006).

[5] S.I. Braginskii, in Reviews of Plasma Physics Ed. M.Leontovich (Consultants Bureau, New York, 1965), Vol.1, p. 205.

[6] B. Cohen, A. Kemp and L. Divol, Simulation of LaserPlasma Interactions and Fast Electron Transport in Inhomogeneous Plasma submitted to J.Comp.Phys. (2009)

[7] C. Birdsall and A.B. Langdon, Plasma Physics via Computer Simulation (Adam Hilger, New York, 1991).

[8] Y Sentoku and A. J Kemp. Numerical methods for particle simulations. Journal of Computational Physics, 227:16, May 2008.
[9] J. Davies, A. R. Bell, M. Haines, and S. Guérin, Shortpulse high-intensity laser-generated fast electron transport into thick solid targets. Physical Review E 56, 7193 (1997).

[10] L. Gremillet, G. Bonnaud, and F. Amiranoff, Filamented transport of laser-generated relativistic electrons penetrating a solid target. Phys Plasmas 9, 941 (2002).

[11] J. J. Honrubia, A. Antonicci, and D. Moreno, Laser and Particle Beams 22, p.129 (2004).

[12] A. J. Kemp, Y. Sentoku, V. Sotnikov, and S. Wilks, Collisional relaxation of superthermal electrons generated by relativistic laser pulses in dense plasma. Phys Rev Lett. 97, 235001 (2006).

[13] A. Kemp and J.Meyer-ter-Vehn. An equation of state code for hot dense matter based on the QEOS description. Nuclear Instruments and Methods in Physics Research Section A, 415:674, Sep 1998.

[14] T. Ma, private communication (October 2009)

[15] T Ma, M. H Key, R. J Mason, K. U Akli, R. L Daskalova, R. R Freeman, J. S Green, K Highbarger, P. A Jaanimagi, J. A King, K. L Lancaster, S. P Hatchett, A. J MacKinnon, A. G Macphee, P. A Norreys, P. K Patel, R. B Stephens, W Theobald, L. D van Woerkom, M. S Wei, S. C Wilks, and F. N Beg. Transport of energy by ultraintense laser-generated electrons in nail-wire targets. 
Phys Plasmas, 16:2702, Nov 2009. 